\title{
Electrocardiographic changes in hypertensive disorders of pregnancy
}

\author{
Fabio Angeli, Enrica Angeli and Paolo Verdecchia
}

Hypertension Research (2014) 37, 973-975; doi:10.1038/hr.2014.128; published online 21 August 2014

$\mathrm{H}$ ypertensive disorders of pregnancy are a major cause of poor pregnancy outcome and complicate $\sim 6-11 \%$ of all pregnancies. ${ }^{1}$ These disorders encompasses a spectrum of conditions, which range in severity from a mild increase in blood pressure (BP) at term with no additional signs/symptoms or adverse sequelae to multisystem conditions with the potential for significant maternal, fetal and neonatal harm. When diagnosed during pregnancy, hypertension disorders increase the maternal risk of placental abruption, organ failure, cerebrovascular accident and disseminated intravascular coagulation, as well as fetal risk of intrauterine growth restriction, intrauterine death and prematurity. ${ }^{2}$

Traditionally, hypertensive disorders during pregnancy are classified into four categories: (a) chronic hypertension, (b) preeclampsia-eclampsia, (c) preeclampsia superimposed on chronic hypertension and (d) gestational hypertension. ${ }^{1}$ Nevertheless, the Society of Obstetricians and Gynecologists of Canada (SOGC) recently released revised guidelines that classified hypertension disorders of pregnancy as pre-existing hypertension, gestational hypertension (with the options to add 'with comorbid conditions' or 'with evidence of preeclampsia' to either category), preeclampsia or 'other hypertensive effects' (transient hypertensive effect, white-coat and masked hypertensive effects). ${ }^{3}$ The latter category was added to raise awareness that office BP that is not consistently elevated may still be

F Angeli is at Division of Cardiology and Cardiovascular Pathophysiology, Hospital 'S.M. della Misericordia', Perugia, Italy; E Angeli is at Division of Obstetrics and Gynecology, Hospital 'San Matteo degli Infermi', Spoleto, Italy; P Verdecchia is at Department of Internal Medicine, Hospital of Assisi, Assisi, Italy

E-mail: fangeli@cardionet.it associated with elevated risks compared with consistently normal BP. ${ }^{4}$

Among the investigations currently used to classify hypertensive disorders of pregnancy (serum creatinine, fasting blood glucose, serum potassium and urinalysis), interpretation of the ECG is an important component in the evaluation of cardiovascular adaptations during pregnancy and it has been recently suggested as a potential tool to identify women at increased risk for the development of these conditions. ${ }^{3}$

\section{NORMAL ADAPTATIONS}

There is a clear suggestion that pregnancy affects the ECG at some time point and that there is restoration of these pregnancyinduced changes late in pregnancy or following delivery (Figure 1).

Heart rate (HR) increases progressively throughout the pregnancy, reaching a peak during the third trimester. This increase in HR seems related to hormonal factors in early stages of pregnancy and later to increased left atrial diameter and sympathetic activation (sinus-node remodelling).

Gestational age also impacts QRS complex and $\mathrm{T}$ waves, promoting a leftward axis shift as pregnancy progresses. In particular, a leftward deviation of the mean QRS axis during the second and third trimesters of pregnancy and then rightward before delivery is observed in the majority of women.

PR interval exhibits a significant reduction in the mean values during pregnancy, ${ }^{5}$ while the QRS amplitude generally increases slightly in the late pregnancy (but without a clear evidence of left ventricular hypertrophy). No clinically significant changes occur in other ECG intervals (including QT interval) or cardiac rhythm (Figure 1).

\section{FEATURES OF INCREASED RISK}

Only a few studies have investigated the ECG changes during normal pregnancy and little is known about ECG alterations during pregnancy-induced hypertensive disorders.

Although little is known about electrophysiological cardiac alterations in pregnant women with hypertensive disorders, there is evidence that hypertensive disorders of pregnancy can be associated with changes in P-wave morphology and QT interval.

Clinical studies have produced conflicting results with regard to QT interval changes and its time course during gestation. The rate-corrected QT interval seems to be unaffected by normal pregnancy, ${ }^{5}$ while pregnancies with abnormal uterine perfusion that developed pathological outcomes (including pre-eclampsia) showed a trend towards shorter QTc intervals compared with pregnant women with normal perfusion. ${ }^{6}$ Interestingly, the QTc interval of women with pathological pregnancy outcomes appeared to shorten progressively throughout the second half of gestation, before the onset of clinical symptoms of hypertension or proteinuria. ${ }^{6}$

On the other hand, Isezuo and Ekele ${ }^{7}$ showed that eclampsia was associated with prolonged ventricular repolarization. Compared to controls, the eclamptics had higher frequency of sinus tachycardia $(9.0 \%$ vs. $13.3 \%)$ and abnormal QTc $(46.7 \%$ vs. 6.6\%, odds ratio (OR): 9.2; 95\% confidence interval (CI): $1.61-68.48, \quad P=0.01)$ as measured on the surface ECG. ${ }^{7}$

For this mixed nature of the evidence, the analysis by Raffaelli et al. ${ }^{8}$ published in the current issue of the Journal adds further data, which need to be combined with the previous literature. 
Briefly, the authors recruited 76 women affected by pre-eclampsia and a control group of pregnant women without cardiovascular disease or gestational hypertension. A standard 12-lead ECG was performed on all patients prior to delivery. ${ }^{8}$

The routine evaluation of the ECGs in both groups was mostly non-pathological. However, pre-eclamptic women showed a lower HR (77.4 \pm 14.3 vs. $81.6 \pm 11.0$ b.p.m.; $P=0.005)$, a longer mean QTc interval (442.7 \pm 26.7 vs. $423.7 \pm 20.7 \mathrm{~ms} ; P<0.001$ ) and a higher QT dispersion (24.0 vs. $22.0 \mathrm{~ms}$; $P<0.001)$ than the control group. ${ }^{8}$

Notably, QT dispersion is an indicator of inhomogeneity in ventricular activity and its prolongation is correlated with an increased incidence of ventricular arrhythmias and is a predictor of all-cause mortality. ${ }^{9}$ Thus, a prolonged QT dispersion could explain the increased incidence of serious ventricular arrhythmias and adverse outcomes in pregnant women with pre-eclampsia.

Nevertheless, assessing the acute cardiac effects of pre-eclampsia on ventricular repolarization, Raffaelli et al. ${ }^{8}$ also documented that treatment with $\mathrm{MgSO}_{4}$ was an independent predictor of prolonged QT interval $(P<0.001)$. Although intriguing, the lack of a formal interaction test between measures of ventricular repolarization and $\mathrm{MgSO}_{4}$ administration does not support the hypothesis that alterations in the QT interval precede clinical manifestations of eclampsia (i.e., the prolonged QTc interval might be only the result of high-dose administration of $\mathrm{MgSO}_{4}$ ). Thus, the notion that increased ventricular repolarization heterogeneity (as documented by higher values of QT dispersion) is a spontaneous high-risk feature of pre-eclampsia for the development of adverse events remains questionable.

More interestingly, the ancillary analysis by Raffaelli et al. ${ }^{8}$ demonstrated that $\mathrm{P}$-wave duration was significantly longer in the pre-eclamptic women than in the control group.

This finding is consistent with a previous prospective screening study from our group, which investigated the potential additive role of standard ECG in the identification of pregnant women at increased risk for hypertensive complications ${ }^{3}$ (Figure 2).

Specifically, 12-lead ECG was recorded at the first antenatal visit and the following ECG parameters were analyzed: HR, QRS duration, QTc interval, Cornell voltage, presence of ST-T abnormalities and left atrial abnormality in lead $\mathrm{V}_{1}$.

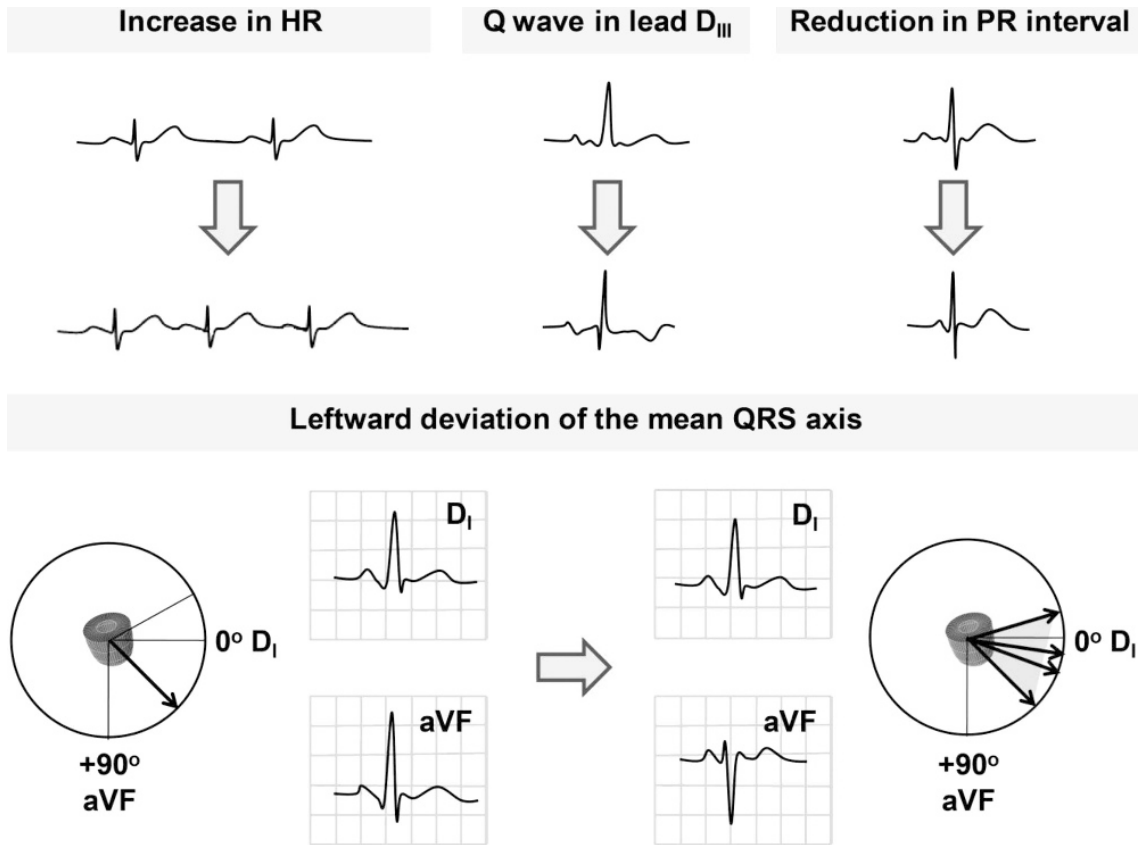

Figure 1 Normal ECG adaptations during pregnancy. ECG changes in normal pregnancy include a reduction in the mean values of $P R$ interval, sinus tachycardia, left axis deviation, inverted or flattened $T$ waves and a $Q$ wave in lead $D_{\| I}$. HR, heart rate.

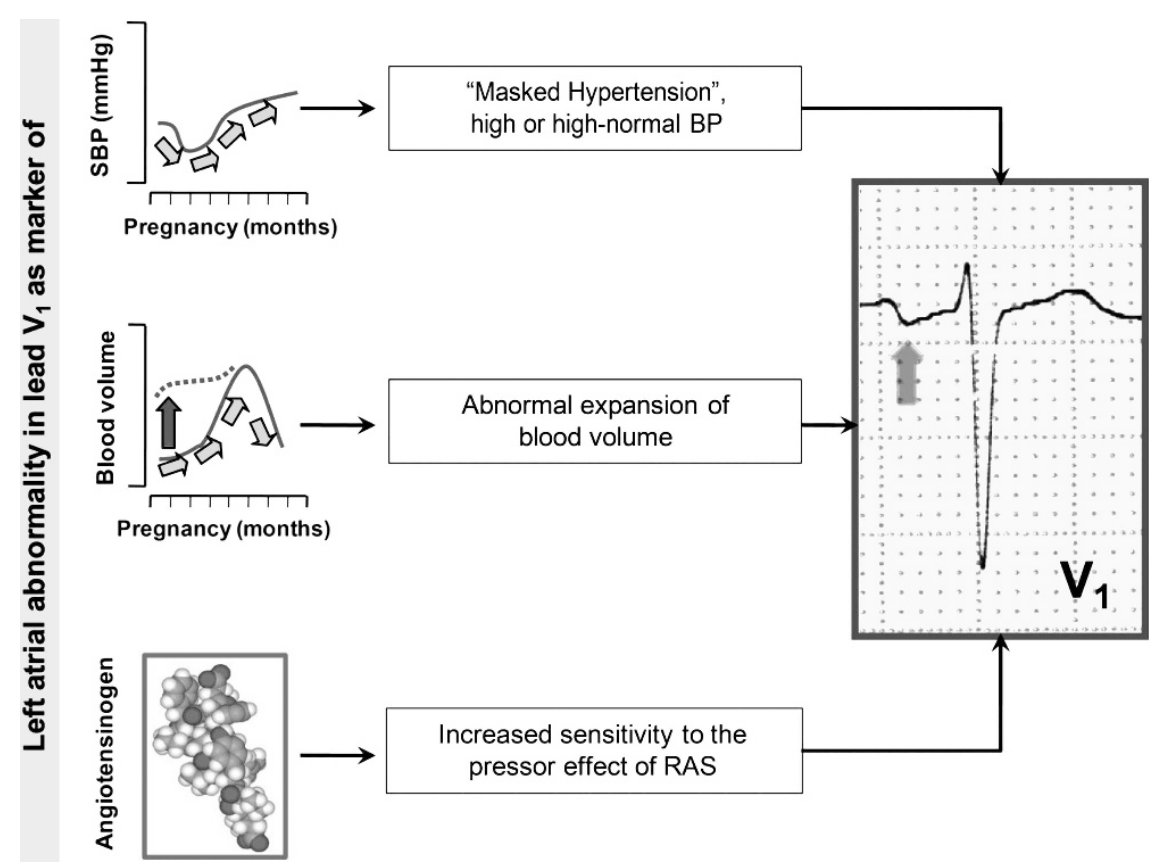

Figure 2 Potential links between left atrial abnormality detected early in pregnancy by ECG and development of hypertensive disorders during pregnancy. Upper panel: the tendency of BP to decrease in early pregnancy may mask the real load of women with abnormal BP before pregnancy. The presence of an abnormal $P$ wave in the early phase of gestation may be considered as a marker of masked hypertension or high or high-normal BP values. Middle panel: expansion of blood volume directly affects left atrial pressure and dimensions; the detection of left atrial (LA) enlargement may be a marker of an abnormal expansion of blood volume in the early phase of pregnancy. Lower panel: in patients developing preeclampsia, there is a loss of the normal pregnancy-associated refractoriness to pressor agents. The increased sensitivity to the pressor effect of the RAS may be involved in the development of LA enlargement. BP, blood pressure; RAS, renin-angiotensin system; SBP, systolic blood pressure. 
The primary outcome of our study was the development of gestational hypertension, pre-eclampsia and eclampsia. The secondary outcome was a composite measure of hypertensive disorders and other pregnancy complications.

In a multivariable model, mean arterial pressure (MAP) and left atrial abnormality were independent predictors of hypertensive disorders. In particular, the presence of left atrial abnormality in lead $V_{1}$ was associated with a fourfold increased risk of developing hypertensive disorders during pregnancy (OR: 4.35; 95\% CI: 1.84-10.31; $P=0.001$ ). The same prediction model was also able to identify pregnant women at increased risk for the occurrence of maternal and fetal/neonatal complications (Figure 2).

\section{PERSPECTIVES}

Pregnancy is physiologically associated with changes in HR, cardiac output and intravascular volume. These changes facilitate the adaptation of the cardiovascular system to the increased metabolic needs of the mother enabling adequate delivery of oxygenated blood to the peripheral tissues and to the fetus. ${ }^{10}$

These alterations in cardiovascular parameters during pregnancy suggest the likelihood of an altered ECG during pregnancy. Typically, ECG changes observed during normal pregnancy include sinus tachycardia, left axis deviation, ectopic beats, inverted or flattened $\mathrm{T}$ waves, a $\mathrm{Q}$ wave in lead $\mathrm{D}_{\mathrm{III}}$ and the augmented voltage unipolar left foot lead ${ }^{5}$ (Figure 1).

The identification of some ECG changes induced by a normal pregnant state helps to establish a reference for comparison. Nevertheless, for many of the clinical manifestations of hypertensive disorders of pregnancy pathological ECG changes have yet to be clearly determined.

In the last few years, particular attention has been placed on changes of $\mathrm{P}$ wave morphology ${ }^{2,3}$ (Figure 2) and QT interval $^{6-8}$ and their modifications during pregnant state. However, clinical studies generated mixed evidence and some findings were based on limited clinical observations. Thus, it is still unclear whether ECG variations are useful to predict hypertensive disorders of pregnancy.

1 Gifford RW, August PA, Cunningham G, Green LA, Lindheimer MD, McNellis D, Roberts JA, Sibai BM, Taler S. Report of the national high blood pressure education program working group on high blood pressure in pregnancy. Am J Obstet Gynecol 2000; 183: S1-S22.

2 Angeli F, Angeli E, Reboldi G, Verdecchia P. Hypertensive disorders during pregnancy: clinical applicability of risk prediction models. J Hypertens 2011; 29: 2320-2323.

3 Angeli E, Verdecchia P, Narducci P, Angeli F. Additive value of standard ecg for the risk prediction of hypertensive disorders during pregnancy. Hypertens Res 2011; 34: 707-713.

4 Magee LA, Pels A, Helewa M, Rey E, von Dadelszen P, Hypertension Guideline C, Magee LA, Audibert F, Bujold E, Cote AM, Douglas MJ, Eastabrook G, Firoz T, Gibson P, Gruslin A, Hutcheon J, Koren G, Lange I, Leduc L, Logan AG, MacDonell KL, Moutquin JM, Sebbag I. Diagnosis, evaluation, and management of the hypertensive disorders of pregnancy: executive summary. J Obstet Gynaecol Can 2014; 36: 416-438.

5 Carruth JE, Mivis SB, Brogan DR, Wenger NK. The electrocardiogram in normal pregnancy. Am Heart $J$ 1981; 102: 1075-1078.

6 Baumert M, Seeck A, Faber R, Nalivaiko E, Voss A. Longitudinal changes in qt interval variability and rate adaptation in pregnancies with normal and abnormal uterine perfusion. Hypertens Res 2010; 33: 555-560.

7 Isezuo SA, Ekele BA. Eclampsia and abnormal qtc. West Afr J Med 2004; 23: 123-127.

8 Raffaelli R, Prioli MA, Parissone F, Prati D, Carli M, Bergamini C, Cacici G, Balestreri D, Vassanelli C, Franchi M. Pre-eclampsia: evidence of altered ventricular repolarization by standard ECG parameters and QT dispersion. Hypertens Res 2014; 37: 984-988.

9 Higham PD, Campbell RW. Qt dispersion. Br Heart J 1994; 71: 508-510.

10 Mahendru AA, Everett TR, McEniery CM, Wilkinson IB, Lees CC. The feasibility of prospectively studying maternal cardiovascular changes from before conception. Hypertens Res 2013; 36: 698-704. 